\title{
Social anxiety disorder co-morbid with schizophrenia: a cross- sectional study from India
}

\author{
Bipeta Rajshekhar ${ }^{1}$, Yerramilli SRR Srinivasa ${ }^{2}$, Lakhan Ram $^{3}$, Khan A Majeed ${ }^{4}$ \\ ${ }^{1}$ Dr. Rajshekhar Bipeta, Consultant Psychiatrist, Rajasri Clinic, Hyderabad, Telangana, India, ${ }^{2}$ Dr. Srinivasa SRR \\ Yerramilli, Consultant Psychiatrist, Sri Venkateswara Nursing Home, Hyderabad, Telangana, India, ${ }^{3}$ Dr. Ram Lakhan, \\ Doctoral Candidate in Epidemiology, School of Public Health Initiative, Jackson State University, USA, ${ }^{4}$ Dr. Majeed A \\ Khan, Consultant Psychiatrist, City Nursing Home, Hyderabad, Telangana, India.
}

Address for Correspondence: Dr Rajshekhar Bipeta, Consultant Psychiatrist, Rajasri clinic, Malkajgiri, Hyderabad500047, Telangana, India. Email: braj111@yahoo.co.in

\begin{abstract}
Introduction: The co-morbidity of various psychiatric disorders with schizophrenia (SZ) is increasingly being recognized, with anxiety disorders (ADs) being no exception. Among the various ADs, the co morbidity of social anxiety disorder (SAD) and SZ is not well studied. We hypothesized that the prevalence of SAD in SZ is high. Objective: We aimed to study the prevalence of SAD in patients with SZ, and to determine the associated socio-demographic and clinical correlates. Materials and methods: This was an outpatient study on consecutively sampled 64 International Diagnostic Criteria (ICD-10) diagnosed treatment-naive SZ patients, who were rated on the Positive and Negative Syndrome Scale (PANSS), the Social Interaction Anxiety Scale (SIAS), the WHO-5 Well-Being Index (WHO-5) and the Global Assessment of Functioning Scale (GAF). Results: The prevalence of SAD in or sample of SZ patients was $26.56 \%$. Compared to the SZ without SAD group, the SZ with SAD group had a lower quality of life (QoL) and GAF scores, but, no significant difference in the PANSS ratings. Conclusions: The SAD is highly co morbid with SZ, and appears to be independent of psychosis, and is associated with lower QoL and psychosocial functioning. Future followup studies should evaluate whether this SAD co-morbidity has any impact on the treatment outcome of SZ.
\end{abstract}

Key words: Co-morbidity; Schizophrenia; Social Anxiety Disorder; Social Phobia

\section{Introduction}

In schizophrenia (SZ) patients, the currently available pharmacological treatments lead to rapid amelioration of positive symptoms. However, the management of negative and cognitive symptoms continue to be challenging. Co-morbid psychiatric disorders are common in SZ; these may worsen the prognosis and have an impact on the psychosocial functioning. The prevalence of anxiety disorders (ADs) in SZ is reported to be $5.4 \%$ to $14.9 \%$ [1]. Among various ADs, the prevalence of social anxiety disorder (SAD) is reported in the range of $11 \%$ to $38 \%$ [2-7].

The International Diagnostic Criteria (ICD-10) diagnostic criteria [8], for social phobia (or social

Manuscript received $4^{\text {th }}$ October 2016

Reviewed: $15^{\text {th }}$ October 2016

Author Corrected: $27^{\text {th }}$ October 2016

Accepted for Publication $17^{\text {th }}$ November 2016 anxiety disorder) are as follows:

"A. Either (1) or (2): 1. Marked fear of being the focus of attention or fear of behaving in a way that will be embarrassing or humiliating. 2. Marked avoidance of being the focus of attention or situations in which there is fear of behaving in an embarrassing or humiliating way.

These fears are manifested in social situations,......

F.Most commonly used exclusion criteria:....not caused by delusions, hallucinations or other symptoms of disorders such as...... schizophrenia and related disorders,..."

In patients with SZ, the shyness and fear of negative evaluation that is characteristic of SAD may be confused with social withdrawal and fear consequent to 
delusions and hallucinations. The symptoms of SAD may go unrecognized in SZ under the disguise of delusions, hallucinations or negative symptoms [7, 9]. Pallanti, et al. [2] reported that in SZ, SAD is independent of paranoia and significantly associated with disability.

However, few of the earlier studies [2, 7] assessed SAD in patients who were already on treatment; thus, treatment could have masked the expression of SAD symptoms. It is imperative to systematically study the prevalence of SAD in treatment naive SZ patients, as this may have therapeutic and prognostic implications.

Based on the earlier work, we hypothesised that SAD is highly prevalent in SZ. We aimed to determine the prevalence of SAD in treatment naive patients with SZ, and study the associated socio-demographic and clinical correlates.

\section{Materials and Methods}

Ours was an outpatient cross-sectional study from an urban psychiatric clinic from India, on treatment seeking and treatment-naive ICD-10 diagnosed [2] schizophrenia patients aged 18 years and above, of both gender; who were consecutively enrolled and purposively sampled.

The patients who were severely ill, or had major medical, organic or substance use disorders, other AxisI disorders (including other anxiety disorders and depressive disorders), below average intelligence, and cognitive impairment were excluded.

The study was conducted as per the ethical standards of the Declaration of Helsinki, 1975 and written informed consent was obtained from the subjects and their legally authorized representatives.

\section{Tools used:}

The Positive and Negative Syndrome Scale (PANSS) [10] is a 30 itemed, seven-point instrument for evaluating the severity of positive, negative and general psychopathology domains in SZ patients.

The Social Interaction Anxiety Scale (SIAS) [11] is a 20-itemed, five-point instrument to assess the prevalence and severity of SAD. A score of 34 or more indicates social phobia.

The WHO-5 Well-Being Index (WHO-5) [12] is a 5item, six point scale to measure the mental well-being of the individual within the previous two weeks, with the higher scores indicating better quality of life (QoL).

The Global Assessment of Functioning scale (GAF) [13] rates the social and occupational functioning of patients from 0 through 100; higher the score better is the functioning.

Statistical analysis: The subjects with SZ were grouped into those with SAD (SZ + SAD) and those without SAD (SZ-SAD).

The unpaired $\mathrm{T}$ test was used for comparing the independent groups and chi-square test was used for comparing the qualitative data. $\mathrm{P}$ value less than 0.05 was considered statistically significant.

A total of 122 patients with SZ were screened; only 64 subjects fulfilled the assessment criteria.

\section{Results}

The final study sample was 64 , and the males predominated $(n=35,54.69 \%)$. The mean age of the subjects was 33.2 years $(\mathrm{SD}+2.28)$.

Table 1 compares the socio-demographic, clinical and rating scores between the SZ+SAD and SZ-SAD groups. Based on a cut-off score of $34 / 60$ on the total SIAS score, $26.56 \%(n=17)$ of our sample was found to have SAD, which was also confirmed on the clinical interview by the psychiatrists.

Thus, the prevalence of SAD in our sample of SZ subjects was $26.56 \%$.

*based on $>34 / 60$ total SIAS score; $\uparrow \mathrm{P}<0.001 ; \ddagger \mathrm{P}>0.05$

Schizophrenia $=$ SZ; social anxiety disorder $=$ SAD; Positive and Negative Syndrome Scale $=$ PANSS; WHO-5 WellBeing Index = WHO-5; Global Assessment of Functioning scale = GAF; Social Interaction Anxiety Scale $=$ SIAS 
Table-1: Comparison of socio-demographic and other parameters between the SZ+SAD and SZ-SAD groups $(\mathrm{N}=64)$.

\begin{tabular}{|c|c|c|c|}
\hline Variable & $\begin{array}{c}\text { SZ + SAD* } \\
(n=17)\end{array}$ & $\begin{array}{c}\text { SZ - SAD* } \\
(n=47)\end{array}$ & Statistical significance \\
\hline & Mean (SD) & Mean (SD) & \\
\hline Age (years) & $31.8+11.2$ & $29.4+10.9$ & $\mathrm{P}=0.443, \mathrm{t}=0.77$ \\
\hline Gender & & & Chi square $=0.938, P=0.333$ \\
\hline Males & 11 & 24 & \\
\hline Females & 6 & 23 & \\
\hline Past history of suicidal attempts & 9 & 5 & Chi-square $=13.073, \mathrm{P}=0.000 \dagger$ \\
\hline \multicolumn{4}{|l|}{ PANSS } \\
\hline PANSS Positive & $31.23+2.21$ & $32.01+1.18$ & $\mathrm{P}=0.074, \mathrm{t}=1.82$ \\
\hline PANSS Negative & $29.19+2.28$ & $29.35+3.21$ & $\mathrm{P}=0.851, \mathrm{t}=0.19$ \\
\hline $\begin{array}{c}\text { PANSS General } \\
\text { psychopathology scale }\end{array}$ & $57.28+4.47$ & $56.19+3.39$ & $\mathrm{P}=0.302, \mathrm{t}=1.04$ \\
\hline PANSS Total & $117.7+10.18$ & $117.55+11.08$ & $\mathrm{P}=0.961, \mathrm{t}=0.05$ \\
\hline WHO-5 & $12.18+1.15$ & $14.35+3.28$ & $\mathrm{P}$ value $=0.010 \ddagger, \mathrm{t}=2.66$ \\
\hline GAF & $22.6+7.31$ & $27.9+9.18$ & $\mathrm{P}$ value $=0.036 \ddagger, \mathrm{t}=2.1437$ \\
\hline
\end{tabular}

\section{Discussion}

The studies assessing co-morbidity of SAD and SZ are sparse. In our sample of 64 treatment-naive patients with SZ, the prevalence of SAD was $26.56 \%$, which was confirmed, both clinically as well as on SIAS. Our rates are in tune with the studies by Lowengrub, et al (38\%) [7], Pallanti et al. (36.3\%) [2], Tibbo et al. (23.3\%) [14] and Braga et al. (17\%) [4]. These variations could be due to the methodological issues, patient selection and use of different rating scales.

Consistent with Pallanti et al. [2] and Lowengrub, et al. [7], we too found lower QoL (WHO-5 scores) in $\mathrm{SZ}+\mathrm{SAD}$ group. However, our SZ+SAD group also had lower GAF scores; while in those studies the difference in GAF scores was not significant. This variation could be because ours was a treatment naive sample; while the subjects in the earlier studies had already sought some treatment probably leading to better social and occupational functioning.

A question worth pondering is whether the SAD symptoms are a function of paranoia or because of the negative symptoms? This issue was addressed by the fact that the PANSS positive and negative scores were not different between the two groups; thus, indicating that the SAD was independent of paranoia and negative symptoms. Thus, we confirmed the findings of earlier studies $[2,7,15]$.
Similar to Pallanti et al. [2], significantly more number of our SZ + SAD subjects attempted suicide in past. This cannot be attributed to depression as this was our exclusion criterion. This higher rate of suicidality could be due to the social deficits in the context of SZ which is further compounded by social anxiety.

The strengths of our study include its naturalistic setting; also, we could assess baseline level of SAD symptoms in treatment-naive SZ patients. This is important because, dopamine antagonists such as clozapine can precipitate SAD symptoms [16], thus making it difficult to ascertain whether SAD is part of $\mathrm{SZ}$ or is drug-induced.

The limitations of our study include its cross-sectional design with no control group; hence, the results cannot be generalized to the community dwelling SZ patients. We used convenience sampling and did not use structured diagnostic schedule for diagnosing SZ and co-morbidities. Also, we could not ascertain which symptoms started first, SAD or psychotic. The SIAS is not standardized for Indian population.

Thus, the SAD co-morbidity may compound the disability, and impair the QoL and psychosocial functioning of patients with SZ. All patients with SZ should be screened and treated for SAD. The drugs such 
as specific serotonin-reuptake inhibitors [17], oxytocin [18] and psychosocial therapies such as cognitive behavioural therapy $[19,20]$ have been used with success.

\section{Conclusion}

The clinicians should be vigilant of the high SZ-SAD co-morbidity. There is a need for prospective studies to further establish this association, and to assess how these patients progress with treatment.

We express our heartfelt gratitude to Prof Majeed A Khan who expired on 10 May 2015. He made significant contribution to the conceptualization and design of this study.

\section{Funding: Nil, Conflict of interest: None. Permission of IRB: Yes}

\section{References}

1. Achim AM, Maziade M, Raymond E, et al. How prevalent are anxiety disorders in schizophrenia? a meta-analysis and critical review on a significant association. Schizophr Bull 2011; 37 (4): 811-821.

2. Pallanti S, Quercioli L, Hollander E. Social anxiety in outpatients with schizophrenia: a relevant cause of disability. Am J Psychiatry. 2004 Jan;161(1):53-8.

3. Voges M, Addington J. The association between social anxiety and social functioning in first episode psychosis. Schizophr Res. 2005 Jul 15;76(2-3):287-92.

4. Braga RJ, Mendlowicz MV, Marrocos RP, Figueira IL. Anxiety disorders in outpatients with schizophrenia: prevalence and impact on the subjective quality of life. J Psychiatr Res. 2005 Jul;39(4):409-14. Epub 2004 Nov 13.

5. Mazeh D, Bodner E, Weizman R, Delayahu Y, Cholostoy A, Martin T, Barak Y. Co-morbid social phobia in schizophrenia. Int J Soc Psychiatry. 2009 May; 55(3):198-202. doi: 10.1177/0020764008093447.

6. Cosoff SJ, Hafner RJ. The prevalence of comorbid anxiety in schizophrenia, schizoaffective disorder and bipolar disorder. Aust NZ J Psychiatry 1998; 32: 67-72.

7. Lowengrub KM, Stryjer R, Birger M, et al. social anxiety disorder comorbid with schizophrenia: the importance of screening for this under recognized and undertreated condition. Isr J Psychiatry Relat Sci 2015; 52 (1): 40-46.

8. World Health Organisation. (1992). International Statistical Classification of Diseases and Related Health Problems, 10th Revision (ICD-10). Geneva: WHO.

9. Blanchard JJ, Mueser KT, Bellack AS. Anhedonia, positive and negative affect, and social functioning in schizophrenia. Schizophr Bull. 1998;24(3):413-24.

10. Kay SR, Fiszbein A, Opler LA. The positive and negative syndrome scale (PANSS) for schizophrenia. Schizophr Bull. 1987;13(2):261-76.

11. Mattick R, Clarke C. Development and validation of measure of Social Phobia Scrutiny Fear and Social Interaction Anxiety. Behav Res Ther 1998; 36:455-70.

12. Bech P. Clinical Psychometrics. Oxford, UK: Wiley Blackwell; 2012.

13. American Psychiatric Association: Diagnostic and Statistical Manual of Mental Disorders, 4th. Washington, DC: American Psychiatric Association, 1994.

14. Tibbo P, Swainson J, Chue P, LeMelledo JM. Prevalence and relationship to delusions and hallucinations of anxiety disorders in schizophrenia. Depress Anxiety 2003; 17: 65-72.

15. Michail M, Birchwood M. Social anxiety disorder in first-episode psychosis: incidence, phenomenology and relationship with paranoia. Br J Psychiatry. 2009 Sep; 195(3):234-41. doi: 10.1192/bjp.bp.108.053124.

16. Pallanti S, Quercioli L, Pazzagli A. Social anxiety and premorbid personality disorders in paranoid schizophrenic patients treated with clozapine. CNS Spectr 2000; 5: 29-43

17. Pallanti S, Quercioli L, Rossi A, Pazzagli A. The emergence of social phobia during clozapine treatment and its response to fluoxetine augmentation. J Clin Psychiatry. 1999 Dec;60(12):819-23.

18. Churchland PS, Winkielman P. Modulating social behavior with oxytocin: how does it work? What does it mean? Horm Behav. 2012 Mar;61(3):392-9. doi: 10. 1016/j.yhbeh.2011.12.003. Epub 2011 Dec 14. 
19. Williams MT, Capozzoli MC, Buckner EV, et al. Cognitive behavioural treatment of social anxiety disorder and comorbid paranoid schizophrenia. Clinical Case Studies 2015; 14 (5): 323-341.
20. Kingsep P, Nathan P, Castle D. Cognitive behavioural group treatment for social anxiety in schizophrenia. Schizophr Res. 2003Sep1;63(1-2):121-9.

\section{How to cite this article?}

Bipeta Rajshekhar, Yerramilli SRR Srinivasa, Lakhan Ram, Khan A Majeed. Social anxiety disorder co-morbid with schizophrenia: a cross-sectional study from India. Int J Med Res Rev 2016;4(11):1953-1957.doi:10.17511/ijmrr. 2016.i11.08. 